\title{
Comparing the Performance Potentials of Singleton and Non-singleton Type-1 and Interval Type-2 Fuzzy Systems in Terms of Sculpting the State Space
}

\author{
Jerry M. Mendel, Life Fellow, IEEE, Ravikiran Chimatapu, Graduate Student Member, IEEE, \\ and Hani Hagras, Fellow, IEEE
}

\begin{abstract}
This paper provides a novel and better understanding of the performance potential of a nonsingleton (NS) fuzzy system over a singleton (S) fuzzy system. It is done by extending sculpting the state space works from $\mathrm{S}$ to NS fuzzification and demonstrating uncertainties about measurements, modeled by NS fuzzification: first, fire more rules more often, manifested by a reduction (increase) in the sizes of first-order rule partitions for those partitions associated with the firing of a smaller (larger) number of rulesthe coarse sculpting of the state space; second, this may lead to an increase or decrease in the number of type-1 (T1) and interval type-2 (IT2) first-order rule partitions, which now contain rule pairs that can never occur for $\mathrm{S}$ fuzzification-a new rule crossover phenomenon - discovered using partition theory; and third, it may lead to a decrease, the same number, or an increase in the number of second-order rule partitions, all of which are system dependentthe fine sculpting of the state space. The authors' conjecture is that it is the additional control of the coarse sculpting of the state space, accomplished by prefiltering and the max-min (or max-product) composition, which provides an NS T1 or IT2 fuzzy system with the potential to outperform an S T1 or IT2 system when measurements are uncertain.
\end{abstract}

Index Terms-Interval type-2 (IT2) fuzzy system, nonsingleton (NS) fuzzifier, rule partitions, sculpting the state space, type-1 (T1) fuzzy system.

\section{INTRODUCTION}

$\mathbf{R}$ ECENTLY, Mendel [1], [2] has explained the performance potential of type-1 (T1), interval type-2 (IT2), and general type-2 rule based fuzzy systems (fuzzy systems, for short) as greater sculpting of the state space. All of this was done for rulebased fuzzy systems that use a singleton (S) fuzzifier. This paper

Manuscript received November 19, 2018; revised March 18, 2019; accepted April 26, 2019. Date of publication May 13, 2019; date of current version April 1, 2020. (Corresponding author: Jerry M. Mendel.)

J. M. Mendel is with the University of Southern California, Los Angeles, CA 90089-2564 USA, and also with the College of Artificial Intelligence, Tianjin Normal University, Tianjin 300384, China (e-mail: mendel@sipi.usc.edu).

R. Chimatapu and H. Hagras are with the Computational Intelligence Centre, School of Computer Science and Electronic Engineering, University of Essex, Colchester CO4 3SQ, U.K. (e-mail: rc16956@essex.ac.uk; hani@essex.ac.uk).

This paper has supplementary downloadable material available at http:// ieeexplore.ieee.org, provided by the authors.

Color versions of one or more of the figures in this paper are available online at http://ieeexplore.ieee.org.

Digital Object Identifier 10.1109/TFUZZ.2019.2916103 extends [1] to rule-based fuzzy systems that use a nonsingleton (NS) fuzzifier.

NS fuzzification is used when the measurements that activate a fuzzy system are imperfect or uncertain (due to measurement noise, sensor imperfections or degradation, etc.). It models such a measurement as a fuzzy number (FN) (defined in Section II-A), so that, regardless of the cause of a measurement's imperfections or uncertainties, they are treated within the framework of fuzzy sets and systems; it was introduced and extensively examined for T1 fuzzy systems by Mouzouris and Mendel [3]-[7] and extended to IT2 fuzzy systems by Liang and Mendel [8]. All of the theoretical results that are reported in these T1 and IT2 papers are included in [9, Chs. 6, 11, and 12] and [10, Chs. 3 and 9].

To the best knowledge of the authors, only a few NS fuzzy system papers appeared between 2001 and 2010, namely [11], [12]-[14], and they were for T1 fuzzy systems. But, beginning in 2011, and continuing through 2019, there has been more interest in both T1 and IT2 NS fuzzy systems, e.g., [15]-[31]. These papers all demonstrate that an NS fuzzy system can outperform an S fuzzy system. But, why does this occur?

It was already demonstrated and explained in [6], [9], [10], and [32], that during the inference process in an NS fuzzy system, the NS fuzzifier acts as a prefilter of the measured value $x^{\prime}$ of a rule antecedent variable $x$, i.e., $x^{\prime} \rightarrow f\left(x^{\prime}\right)$. To date, prefiltering is the only explanation for the improved performance due to NS fuzzification.

The goal of this paper is to provide further understanding of the performance improvement potential of an NS fuzzy system over an $\mathrm{S}$ fuzzy system because it is only if such performance improvement potential exists should one even consider using an NS fuzzy system. This goal is accomplished herein by providing a new and novel additional explanation for the improved performance in terms of the sculpting of the state space due to NS fuzzification. The author's conjecture is that it is the additional control of the coarse sculpting of the state space, accomplished by prefiltering and the max-min (or max-product) composition, which provides an NS T1 or IT2 fuzzy system with the potential to outperform an S T1 or IT2 fuzzy system when measurements are uncertain.

This paper assumes that readers are familiar with T1 and IT2 fuzzy sets and systems, and first-and second-order rule partitions, as explained in [1, Sec. III]. 


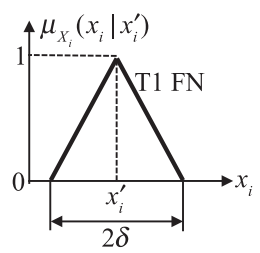

Fig. 1. T1 FN used in this paper.

\section{BACKGROUND}

\section{A. NS Fuzzifiers}

Recall that, for an IF-THEN rule $^{1}$ with $p$ antecedents, $\mathbf{x}=\left(x_{1}, \ldots, x_{p}\right)^{T} \in X_{1} \times X_{2} \times \cdots \times X_{p} \equiv \mathbf{X}$, in a T1 fuzzy system, the fuzzifier maps $\mathrm{x}=\mathrm{x}^{\prime}$ into a T1 fuzzy set (FS) $A_{\mathrm{x}^{\prime}}$ in X. A T1 NS fuzzifier maps measurement $x_{i}=x_{i}^{\prime}$ into a T1 fuzzy number $(\mathrm{FN})^{2}$ for which $\mu_{X_{i}}\left(x_{i}^{\prime}\right)=1$ and $\mu_{X_{i}}\left(x_{i}\right)$ decreases from unity as $x_{i}$ moves away from $x_{i}^{\prime}$ and is denoted $\mu_{X_{i}}\left(x_{i} \mid x_{i}^{\prime}\right)$ (e.g., the T1 FN in Fig. 1).

For an IT2 fuzzy system, the fuzzifier maps $\mathbf{x}=\mathbf{x}^{\prime}$ into an IT2 FS $\tilde{A}_{\mathrm{x}^{\prime}}$ in $\mathbf{X}$, and two kinds of NS fuzzifiers are possible, T1 NS and IT2 NS. In this paper, results are provided only for the T1 NS fuzzifier because an understanding of NS fuzzification in an IT2 fuzzy system, in terms of sculpting the state space, can be accomplished by examining it only for the T1 NS situation (see Section VII in the SM for verification of this).

Additionally, in this paper, it is assumed that all variables are normalized to $[0,10]$, and most examples are provided for (see Fig. 1) $2 \delta=4 \%$ and $12 \%$ of 10 . Some examples are also provided for $2 \delta=24 \%$ of 10 .

\section{B. Firing Level (Interval) in an NS T1 (IT2) Fuzzy System}

It is well known that, for an NS T1 (IT2) fuzzifier, when $\mathbf{x}=\mathbf{x}^{\prime}$ the firing level (interval) $f^{l}\left(\mathbf{x}^{\prime}\right)\left(\left[f^{l}\left(\mathbf{x}^{\prime}\right), \bar{f}^{l}\left(\mathbf{x}^{\prime}\right)\right]\right)$ for each rule $(l=1, \ldots, M)$ is (e.g., [6], [8], [9, Chs. 6, 11, 12], and $[10, \text { Chs. } 3 \text { and 9] })^{3}$

NS T1 fuzzy system

$$
f^{l}\left(\mathbf{x}^{\prime}\right)=T_{i=1}^{p} f^{l}\left(x_{i}^{\prime}\right)=T_{i=1}^{p} \max _{x_{i} \in X_{i}} \mu_{X_{i}}\left(x_{i} \mid x_{i}^{\prime}\right) \star \mu_{F_{i}^{l}}\left(x_{i}\right)
$$

\section{T1 NS IT2 fuzzy system}

$$
\left\{\begin{array}{l}
{\left[f_{-}^{l}\left(\mathbf{x}^{\prime}\right), \bar{f}^{l}\left(\mathbf{x}^{\prime}\right)\right]=\left[T_{i=1}^{p} f_{-}^{l}\left(x_{i}^{\prime}\right), T_{i=1}^{p} \bar{f}^{l}\left(x_{i}^{\prime}\right)\right]} \\
f_{-}^{l}\left(x_{i}^{\prime}\right)=\max _{x_{i} \in X_{i}} \mu_{X_{i}}\left(x_{i} \mid x_{i}^{\prime}\right) \star \mu_{\tilde{F}_{i}^{l}}\left(x_{i}\right) . \\
\bar{f}^{l}\left(x_{i}^{\prime}\right)=\max _{x_{i} \in X_{i}} \mu_{X_{i}}\left(x_{i} \mid x_{i}^{\prime}\right) \star \bar{\mu}_{\tilde{F}_{i}^{l}}\left(x_{i}\right)
\end{array} .\right.
$$

\footnotetext{
${ }^{1}$ For the structures of T1 and IT2 rules (which should be familiar to the readers of this paper), see Section $\mathrm{I}$ in the supplementary material (SM).

${ }^{2}$ Although there are different definitions of a T1 FN, in this paper a fuzzy set $A$ in $R$ is called a $T 1 F N$ if: (1) $A$ is normal, (2) $A$ is convex, and (3) $A$ has bounded support. If a Gaussian MF is used then it is assumed that such a MF is truncated, so as to satisfy condition (3).

${ }^{3}$ Many times (1) and (2) are stated using "sup" instead of "max." For all of the membership functions (MFs) considered in this paper, the sup and max are the same.
}

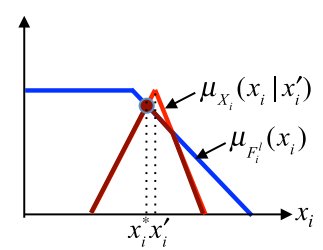

(a)

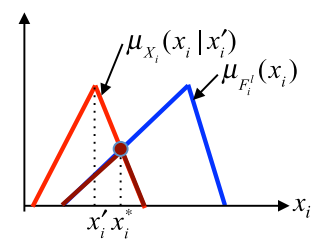

(b)
Fig. 2. Examples of the max-min calculation (cardinal figure); $x_{i}^{*}$ (filled-in cardinal circle) occurs to the (a) left of $x_{i}^{\prime}$ and (b) right of $x_{i}^{\prime}$.

In these equations, ${ }^{4} T$ and $\star$ denote $t$-norms, usually chosen to be the same, either as the product or the minimum, under-barred (over-barred) quantities denote lower (upper) MFs [LMFs (UMFs)], and $F_{i}^{l}\left(\tilde{F}_{i}^{l}\right)$ denotes the T1 (IT2) FS for the $i$ th antecedent in the $l$ th rule. In the sequel, $x_{i}^{*}$ denotes the value of $x_{i}$ at which the maximum occurs in (1) [or, in each of the two lines of (2)].

Examples of max-min calculations between two T1 FSs are depicted in Fig. 2 and are included to remind the reader of the geometry of these calculations. Going down (up) an incline, $x_{i}^{*}$ lags (leads) $x^{\prime}$. Formulas for $x_{i}^{*}$ are given in Table SM-1 of the $\mathrm{SM}$ for both the minimum and product $t$-norms.

Definition 1: $\operatorname{In}^{5}$ a fuzzy system, a firing level (interval) is said to contribute to its output only if it is nonzero. In an NS T1 (T1 NS IT2) fuzzy system, this occurs when $(i=1, \ldots, p) \max _{x_{i} \in X_{i}} \mu_{X_{i}}\left(x_{i} \mid x_{i}^{\prime}\right) \star \mu_{F_{i}^{l}}\left(x_{i}\right)$ $\left(\max _{x_{i} \in X_{i}} \mu_{X_{i}}\left(x_{i} \mid x_{i}^{\prime}\right) \star \bar{\mu}_{\tilde{F}_{i}^{l}}\left(x_{i}\right)\right)$ is simultaneously nonzero for all $p$ antecedents. ${ }^{6}$

\section{Rule Partitions in an S Fuzzy System}

The work in [1, Sec. III] explains that in an S T1 or S IT2 fuzzy system, when a firing level (interval) is computed using either the minimum or product $t$-norms, then its nonzero occurrence over $X_{1} \times X_{2} \times \cdots \times X_{p}$ can be established by examining the components of the firing level (interval) separately over each $X_{i}$ and then combining those results for all $i=1, \ldots, p$, by using either the minimum or product $t$-norms.

Definition 2: [1] In a ${ }^{7}$ T1 (IT2) fuzzy system, a T1 (IT2) first-order rule partition of $X_{i}$ is a collection of nonoverlapping intervals in $X_{i}$, in each of which the same number of same rules is fired whose firing levels (intervals) contribute to the output of that system.

Definition 3: [1] In an S T1 (S IT2) fuzzy system, a T1 (IT2) second-order rule partition [line] of $X_{i}$ occurs where the [slope $^{8}$ of the] MF (LMF or UMF) of a T1 (IT2) fuzzy set that

\footnotetext{
${ }^{4}$ For an explanation of why these equations are valid for both Mamdani and Takagi-Sugeno-Kang (TSK) fuzzy systems, see Section II in the SM.

${ }^{5}$ This is a generalization of [1, Def. 7] from S to NS fuzzy systems.

${ }^{6}$ If the UMF is zero then the LMF must also be zero because an LMF can never be larger than a UMF.

${ }^{7}$ The prefix " $\mathrm{S}$ " is omitted here because, importantly, this definition does not depend upon the nature of the fuzzifier.

${ }^{8}$ Usually, when the MF (footprint of uncertainty-FOU) of a T1 (IT2) fuzzy set that is associated with $x_{i}$ changes its mathematical formula, the slope (derivative) of the MF (LMF or UMF) changes. Using "slope of the" accommodates, e.g., a Gaussian MF whose formula does not change, but whose slope changes at its center of gravity.
} 


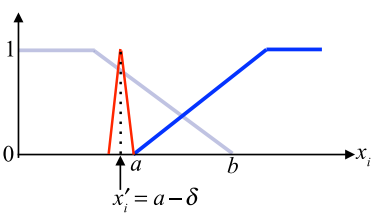

(a)

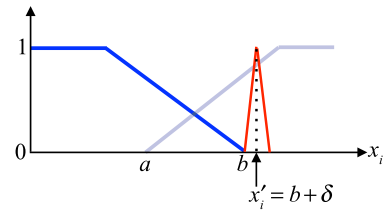

(b)
Fig. 3. (a) First encounter. (b) Last encounter.

is associated with $x_{i}$ changes its mathematical formula within a T1 (IT2) first-order rule partition of $X_{i}$.

Definition 4: [2] Points at which the MF (LMF or UMF) change their mathematical formula (slope) are called MF kinks. In this paper, to keep things relatively simple, it is assumed that such kinks only occur when a membership grade is unity or zero.

Rule partitions sculpt the state space into hyper-rectangles within each of which resides a different nonlinear function (which is why a rule-based fuzzy system is a variable-structure system). First-order rule partitions provide a course sculpting whereas second-order rule partitions provide fine sculpting. To remind the reader, [1] shows that

“... an S T1 fuzzy system can sculpt its state space with greater variability than a crisp rule-based system can, and in ways that cannot be accomplished by the crisp system, and an S IT2 fuzzy system (that has the same number of rules as the S T1 fuzzy system) can sculpt the state space with even greater variability, and in ways that cannot be accomplished by an $\mathrm{S}$ T1 fuzzy system" (and the latter can occur even when S T1 and S IT2 fuzzy systems are described by the same number of parameters).

Many examples of first- and second-order rule partitions for S T1 and S IT2 fuzzy systems are in [1] and its SM. ${ }^{9}$

\section{RULE PARTITIONS FOR NS FUZZY Systems}

This section defines and illustrates first-and second-order rule partitions for NS fuzzy systems because they will help us to further understand what is happening in a fuzzy system as one goes from S to NS fuzzification.

\section{A. First-Order Rule Partitions in an NS Fuzzy System}

1) First-Order Rule Partitions for Each $X_{i}$ : Definition 2 is valid for both S and NS fuzzy systems.

Definition 5: In an NS T1 (T1 NS IT2) fuzzy system, the first encounter [see Fig. 3(a)] between a T1 FN and an upwardsloping MF (UMF or LMF) occurs along the $x_{i}$-axis when $x_{i}^{\prime} \in X_{i}$ is $\delta$-units to the left of where the leading edge of the T1 FN first meets the upward-sloping MF (UMF or LMF), which is at an MF kink. It is just to the right of the first encounter that (1) $[(2)]$ is nonzero for the first time.

Definition 6: In an NS T1 (T1 NS IT2) fuzzy system, the last encounter [see Fig. 3(b)] between a T1 FN and a downwardsloping MF (UMF or LMF) occurs along the $x_{i}$-axis when

\footnotetext{
${ }^{9}$ For the convenience of the reader, Section IV of the SM to this paper contains six tables from [1] that provide notations used in first-and second-order rule partitions as well as procedures for establishing them.
}

TABLE I

ThreE-STEP PROCEDURE FOR ESTABLISHING T1 (IT2) FIRST-ORDER RULE PARTITION QUANTITIES FOR $X_{i}$, IN AN NS T1 (T1 NS IT2) FUZZY SYSTEM

\begin{tabular}{ll}
\hline \hline Step & \multicolumn{1}{c}{ Description } \\
\hline 1 & $\begin{array}{l}\text { Start with a first-order rule partition diagram for the S T1 (IT2) } \\
\text { fuzzy system (see Table SM-III in the SM) }\end{array}$ \\
& Insert (green) $2 \delta$-bands centered at appropriate locations for \\
& first-order rule partition lines (Def. 7) on the $x_{i}$ axis. \\
& Shift the first-order partition lines to the mid-points of their \\
& respective $2 \delta$-bands. Do not do this for the dashed vertical lines \\
& from Step 1 that occur at the start and end ${ }^{\text {a }}$ of $X_{i}$. The shifted \\
& lines are the first-order rule partition lines for the NS T1 (T1 NS \\
& IT2) fuzzy system
\end{tabular}

${ }^{\text {a } I f ~ t h e y ~ w e r e ~ m o v e d ~ t h e y ~ w o u l d ~ f a l l ~ o u t s i d e ~ o f ~} X_{i}$, which is not permissible.

$x_{i}^{\prime} \in X_{i}$ is $\delta$-units to the right of where the lagging edge of the T1 FN last meets the downward-sloping MF (UMF or LMF), which is also at an MF kink. It is just to the left of the last encounter that (1) [(2)] is nonzero for the last time.

Definitions 5 and 6 lead to the following mnemonics: upward sloping left (USL) and downward sloping right (DSR).

Definition 7: Appropriate locations for T1 (IT2) first-order rule partition lines are on the $x_{i}$-axis, and in an NS T1 (T1 NS IT2) fuzzy system they are found by locating all first encounters of upward-sloping MF (UMF) lines and all last encounters of downward-sloping MF (UMF) lines.

Note that a UMF is always reached before an LMF is reached, and so UMFs play an exclusive role in establishing IT2 firstorder rule partitions; however, as will be seen below, UMFs and LMFs both play important roles in establishing IT2 secondorder rule partitions.

A formal three-step procedure for establishing first-order rule partition quantities in an NS fuzzy system for a single variable $x_{i}$ is given in Table I. It is the continuation of the procedure for the respective $\mathrm{S}$ fuzzy system.

Example 1: Consider $x_{i} \in[0,10]$ covered by the three $\mathrm{T} 1$ FSs depicted in Fig. 4(a) (Step 1 in Table I), for which there are three T1 rules whose antecedents are $R^{1}: \mathrm{IF} x_{1}$ is $L$, $R^{2}:$ IF $x_{1}$ is $M$ and $R^{3}:$ IF $x_{1}$ is $H$, and five T1 first-order rule partitions. The results for Steps 2 and 3 in Table I are shown in Fig. 4(b) and (c) for the 4\% T1 FN and 12\% T1 FN, respectively; these figures show the $2 \delta$-bands as well as the shifted partition lines (Steps 2 and 3), which occur at the appropriate locations $x_{i}=a-\delta, b+\delta, c-\delta$, and $d+\delta$.

Comparing Fig. 4(a)-(c), we observe the following:

1) The widths of the one fired-rule $\mathrm{T} 1$ first-order rule partitions (1, 3, and 5) are smaller for NS fuzzification than for S fuzzification.

2) The widths of the two fired-rule T1 first-order rule partitions (2 and 4) are larger for NS fuzzification than for S fuzzification.

3) As the $\mathrm{T} 1 \mathrm{FN}$ goes from $4 \%$ to $12 \%$, the widths of the $\mathrm{T} 1$ one-rule partitions get smaller and smaller, whereas the widths of the T1 two-rule partitions get larger and larger.

This example reveals that in an NS T1 fuzzy system, uncertainties about measurements, modeled by NS fuzzification, fires more rules more often. 


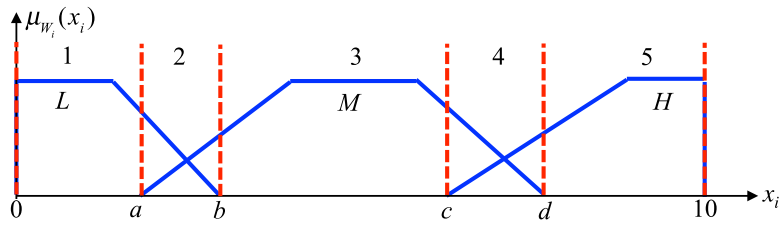

(a)

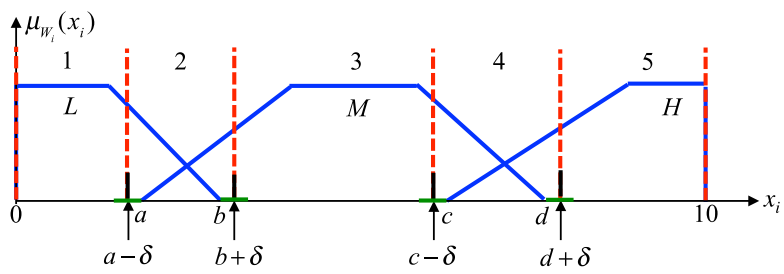

(b)

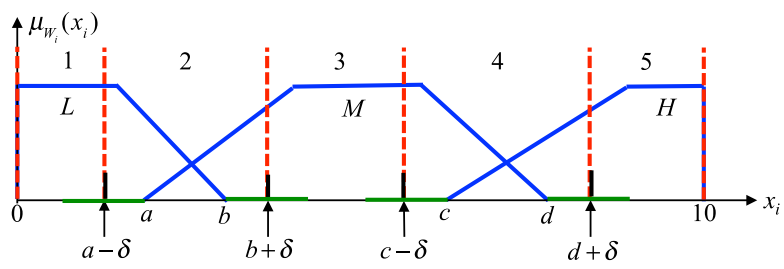

(c)

Fig. 4. Example 1 figures. Numbers above MFs denote numerical names for the five T1 first-order rule partitions (denoted $P_{T 1}^{1}\left(k_{x_{i}} \mid x_{i}\right)$ in [1] where $\left.k_{x_{i}}=1, \ldots, 5\right)$; see also Table SM-II in the SM. (a) S T1. (b) NS T1 (4\% T1 FN). (c) NS T1 (12\% T1 FN).

Example 2: Next, all of the T1 FSs in Example 1 and Fig. 4 are replaced by the IT2 FSs in Fig. 5, whose FOUs were constructed so that the T1 MFs in Fig. 4 are blurred as follows: $a \in\left[a^{\prime}, e^{\prime}\right], b \in\left[f^{\prime}, b^{\prime}\right], c \in\left[c^{\prime}, g^{\prime}\right]$, and $d \in\left[h^{\prime}, d^{\prime}\right]$. Fig. 5(a) is for Step 1 in Table I, whereas Fig. 5(b) and (c) are for the combined Steps 2 and 3 in it.

Comparing the results in Figs. 4 and 5, we observe that the widths of one fired-rule IT2 first-order rule partitions (1, 3, and 5 ) are smaller than those of analogous $\mathrm{T} 1$ first-order rule partitions; and widths of two fired-rule IT2 first-order rule partitions ( 2 and 4) are larger than those of analogous T1 first-order rule partitions. See, also, Example SM-1 in Section VI of the SM.

Generally speaking: uncertainty from T1 NS fuzzification or antecedent MF uncertainty (modeled as an FOU) reduces sizes of a fewer number of fired-rule first-order rule partitions and increases sizes of a greater number of fired-rule first-order rule partitions.

2) First-Order Rule Partitions for $X_{1} \times X_{2}$ :

Definition 8: In an NS T1 (T1 NS IT2) fuzzy system, a T1 (IT2) first-order rule partition of $X_{1} \times X_{2}$ is a collection of non-over-lapping rectangles (squares) of ${ }^{10} X_{1} \times X_{2}$, in each of which the same number of same rules is fired whose firing levels (intervals) contribute to the output of that system.

This definition is unchanged from the one that is given for an $\mathrm{S}$ T1 fuzzy system in [1, Def. 10].

On a drawing of the MFs (FOUs) of $x_{1}$ on the horizontal axis and MFs (FOUs) of $x_{2}$ on the vertical axis, a formal four-step

\footnotetext{
${ }^{10}$ As in [1, footnote 9], if a rule has $p$ antecedents, then $X_{1}$ and $X_{2}$ each denote the universe of discourse for any two of them.
}

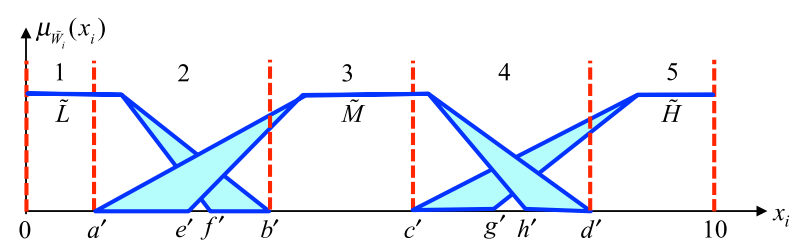

(a)

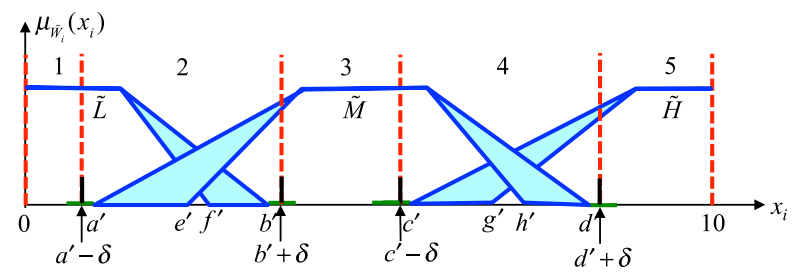

(b)

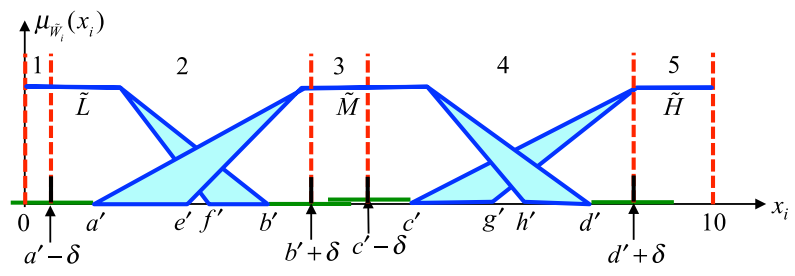

(c)

Fig. 5. Example 2 figures. Numbers above FOUs denote numerical names for the five IT2 first-order rule partitions (denoted $P_{I T 2}^{1}\left(k_{x_{i}} \mid x_{i}\right)$ in [1], where $\left.k_{x_{i}}=1, \ldots, 5\right)$; see also Table SM-II in the SM. (a) S IT2. (b) T1 NS IT2 $(4 \%$ T1 FN). (c) T1 NS IT2 (12\% T1 FN).

TABLE II

FOUR-STEP PROCEDURE FOR ESTABLISHING T1 (IT2) FIRST-ORDER RULE PARTITION QUANTITIES FOR $X_{1} \times X_{2}$ IN AN NS T1 (T1 NS IT2) FUZZY SYSTEM

\begin{tabular}{|c|c|}
\hline Step & Description \\
\hline 1 & $\begin{array}{l}\text { Locate T1 (IT2) first-order rule partitions of } X_{1}\left(X_{2}\right) \text { on the } \\
\text { horizontal (vertical) axis, and establish the number of rules in each } \\
\text { partition and also the total number of such partitions for } X_{1}\left(X_{2}\right)\end{array}$ \\
\hline 2 & $\begin{array}{l}\text { Extend all dashed T1 (IT2) first-order rule partitions (turning them } \\
\text { into solid lines) so that they cover } X_{1} \times X_{2} \text {. The results from } \\
\text { doing this will be a collection of rectangles (or squares) }\end{array}$ \\
\hline 3 & $\begin{array}{l}\text { Compute the fixed number of rules fired in each T1 (IT2) first- } \\
\text { order rule partition using (4) for } p=2\end{array}$ \\
\hline 4 & $\begin{array}{l}\text { Compute the total number of T1 (IT2) first-order rule partitions of } \\
X_{1} \times X_{2} \text { using (3) for } p=2\end{array}$ \\
\hline
\end{tabular}

procedure for establishing T1 (IT2) first-order rule partitions of $X_{1} \times X_{2}$ is given in ${ }^{11}$ Table II. In order to implement this procedure, one must first complete the Table I four-step procedure for establishing the T1 (IT2) first-order rule partitions for $X_{1}$ and $X_{2}$.

Example 3: This is an extension of Examples 1 and 2 from one to two variables in which $x_{1}, x_{2} \in[0,10]$ and both variables are covered by three MFs (FOUs), which are depicted in Figs. 4(a) and 5(a) for which there are now nine rules, whose antecedents for the T1 rules are: $R^{1}\left(R^{2}, R^{3}\right): \operatorname{IF} x_{1}$ is $L$ and $x_{2}$

\footnotetext{
${ }^{11}$ Table II is very similar to Table III in [1] (Table SM-IV in SM), but does not have the symbols that are in the latter (the definitions of which are in $[1$, Table I] or Table SM-II in SM).
} 


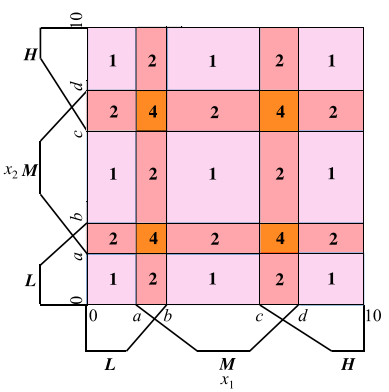

(a)

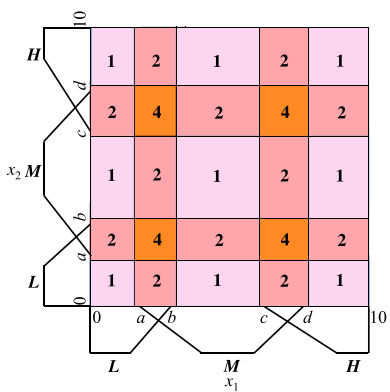

(c)

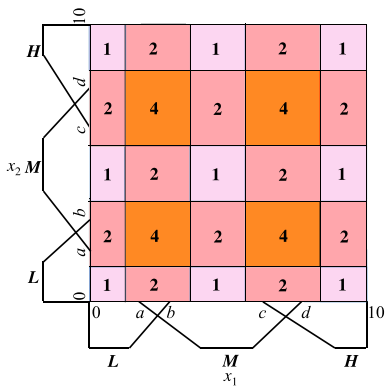

(e)

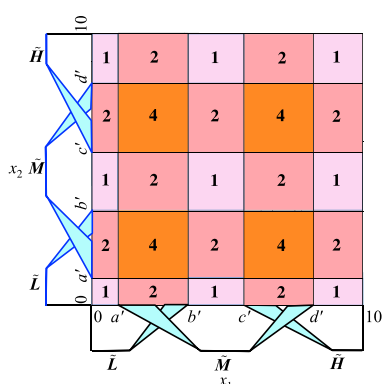

(b)

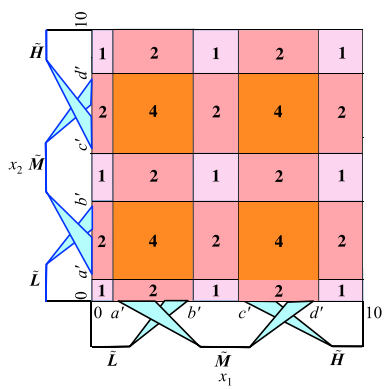

(d)

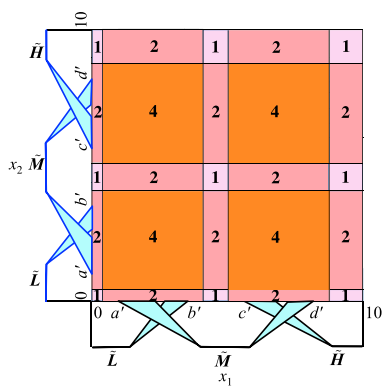

(f)
Fig. 6. Example 3 figures for Table II's Steps 1-3: The number in each rectangle is the number of rules that are fired in it. ${ }^{12}$ (a) S T1. (b) S IT2. (c) NS T1 (4\% T1 FN). (d) T1 NS IT2. (4\% T1 FN). (e) NS T1 (12\% T1 FN). (f) T1 NS IT2 (12\% T1 FN).

is $L(M, H), R^{4}\left(R^{5}, R^{6}\right):$ IF $x_{1}$ is $M$ and $x_{2}$ is $L(M, H)$, and $R^{7}\left(R^{8}, R^{9}\right)$ : IF $x_{1}$ is $H$ and $x_{2}$ is $L(M, H)$. For IT2 rules, replace $L, M, H$ by $\tilde{L}, \tilde{M}, \tilde{H}$. Results for Steps $1-3$ in Table II are shown in the six panels of Fig. 6.

The following is observed from these six figures:

1) Total number of T1 (IT2) first-order rule partitions ${ }^{13}$ on $[0,10] \times[0,10]$ is 25 regardless of the kind of fuzzification.

2) Uncertainty from NS fuzzification reduces sizes of T1 (IT2) first-order rule partitions and increases sizes of T1 (IT2) two- and four-rule partitions.

3) Uncertainty in going from T1 to IT2 FSs also reduces sizes of T1 (IT2) first-order rule partitions and increases sizes of T1 (IT2) two- and four-rule partitions.

\footnotetext{
${ }^{12}$ In [1], $N_{R}\left(k_{x_{1}}, k_{x_{2}}\right)$ denotes this (see, also, Table SM-II in SM), where $k_{x_{1}}, k_{x_{2}}=1, \ldots, 5$ begin in the lowest left-hand square and sweep upwards lexicographically from left to right.

${ }^{13}$ In [1], $N_{*}^{1}\left(X_{1}, X_{2}\right)$ denotes this count (see, also, Table SM-II in SM), where $*=\mathrm{T} 1$ (IT2) for a T1 (IT2) fuzzy system.
}

4) Combined uncertainties from both NS fuzzification and going from T1 to IT2 FSs always leads to largest reductions in sizes of one-rule T1 (IT2) first-order rule partitions and increases in sizes of two- and four-rule T1 (IT2) first-order rule partitions.

NS fuzzification can be said to act as "handles" on the sides of the first-order rule partitions of an S fuzzy system, making the widths of such one (two and four) fired-rule partitions smaller (larger), further confirming that uncertainties about measurements, modeled by NS fuzzification, fires more rules more often.

3) First-Order Rule Partitions for $X_{1} \times X_{2} \times \cdots \times X_{p}$ :

Definition 9: [1] In a T1 (IT2) fuzzy system, a T1 (IT2) first-order rule partition of $X_{1} \times X_{2} \times \cdots \times X_{p}$ is a collection of nonoverlapping hyper-rectangles (or squares) in $X_{1} \times X_{2}$ $\times \cdots \times X_{p}$, in each of which the same number of same rules is fired whose firing levels (intervals) contribute to the output of a T1 (IT2) fuzzy system.

This definition of a T1 (IT2) first-order rule partition of $X_{1} \times X_{2} \times \cdots \times X_{p}$ is the same for $\mathrm{S}$ and NS fuzzy systems.

Although it is impractical (impossible) to use graphical techniques to establish T1 (IT2) first-order rule partitions for $p=3$ $(p \geq 4)$, it is still possible to compute their total number $\left(N_{*}^{1}\right)$, as well as the fixed number of rules that are fired in each hyperrectangle $\left(N_{R}\right)$, by using (6) and (7), respectively, from [1]. For the convenience of the reader, those equations are

$$
\begin{aligned}
N_{*}^{1}\left(X_{1}, X_{2}, \ldots, X_{p}\right) & =\prod_{i=1}^{p} N_{*}^{1}\left(X_{i}\right) \\
N_{R}\left(k_{x_{1}}, k_{x_{2}}, \ldots, k_{x_{p}}\right) & =\prod_{i=1}^{p} N_{R}\left(k_{x_{i}}\right) .
\end{aligned}
$$

Importantly, observe that both $N_{R}$ and $N_{*}^{1}$ are determined for $p \geq 2$ by first determining them for each variable, which is easy to do by the means of the graphical techniques explained above. It is clear, from (3), that as $p$ increases the total number of $\mathrm{T} 1$ (IT2) first-order rule partitions increase dramatically in both T1 and IT2 S and NS fuzzy systems. It is what goes on in each of those partitions that is different for S and NS fuzzy systemsmore rules fire for more of the time in NS fuzzy systems.

\section{B. Second-Order Rule Partitions in an NS Fuzzy System}

1) Second-Order Rule Partitions for Each $X_{i}$ : For NS fuzzification, Definition 3 changes to the following.

Definition 3NS: In an NS T1 (T1 NS IT2) fuzzy system, a $T 1$ (IT2) second-order rule partition line of $X_{i}$ occurs where the location of the value of $x_{i}$ at which the maximum occurs in (1) $[(2)]\left(x_{i}^{*}\right)$ changes from one segment of an antecedent's MF (UMF or LMF) to another segment [within a T1 (IT2) firstorder rule partition of $X_{i}$ ], where the slope of the latter segment differs from the slope of the former segment.

Such a location is easy to visualize for minimum $t$-norm but is more difficult to locate for product $t$-norm, and since the purpose of this paper is to develop a further explanation of what is happening for NS fuzzification in terms of sculpting the state space; here, we only consider the minimum $t$-norm. 


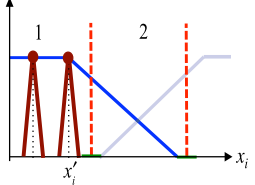

(a)

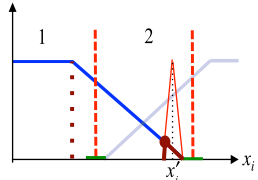

(d)

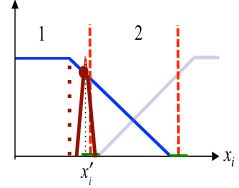

(b)

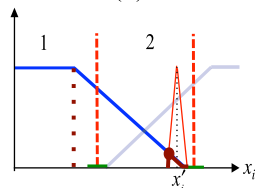

(e)

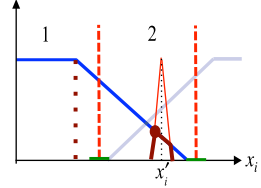

(c)

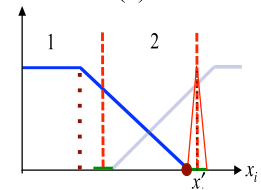

(f)
Fig. 7. Example 4 figures: six locations of a triangle $\mathrm{T} 1 \mathrm{FN}$ in relation to a downward-sloping portion of a left-shoulder MF. In each figure, the dark cardinal piecewise-linear function is the result of the min computation (in the max-min computation) between the T1 FN and the left-shoulder MF, and the cardinal filled-in dot is the result of the max computation.

Note that, for $\mathrm{S}$ fuzzification, $x_{i}^{*}=x_{i}^{\prime}$, so that Definition $3 \mathrm{NS}$ becomes equivalent to Definition 3.

Example 4: Fig. 7 depicts six locations of a triangle T1 FN in relation to the downward-sloping portion of a left-shoulder MF. Observe the flow of the max-min computation, which has to be performed over the entire domain of the $\mathrm{T} 1 \mathrm{FN}$, as the triangle T1 FN moves from left to right.

In Fig. 7(a), the T1 FN (shown at two locations) only intersects the shoulder when $x_{i}=x_{i}^{\prime}$; this continues until the T1 FN reaches the shoulder breakpoint (which is an MF kink at unity membership grade, which is then shown as a dotted secondorder partition line in successive figures). In Fig. 7(b) and (c), the min computation leads to a three-sided cardinal figure, and the result of the max-min computation occurs at the upper lefthand vertex of that figure, which is on the downward-sloping portion of the MF. The projection of that result onto the $x_{i}$ axis would locate $x_{i}^{*}$.

In Fig. 7(d) the leading edge of the T1 FN intersects the downward-sloping portion of the left shoulder MF at zero membership grade (an MF kink), so that the result of the min computation is a triangle; however, the result of the max-min computation is still on the downward-sloping portion of the MF.

In Fig. 7(e), when the T1 FN moves to the right of its location in Fig. 7(d), the result of the max-min computation is still on the downward-sloping portion of the MF.

Finally, in Fig. 7(f), when the top of the left leg of the T1 FN reaches the right-hand boundary of T1 first-order rule Partition \#2 (the second red dashed line), the result of the max-min computation is zero and that ends the analysis of the max-min composition of the triangle T1 FN with the left shoulder MF. Example 5 below continues this example.

The results from this example are summarized in the following.

Definition 10: Appropriate locations for T1 second-order rule partition lines in an NS T1 fuzzy system (that uses a triangle T1 FN) are on the $x_{i}$-axis and are found by locating where an MF has an MF kink (Definition 4) at unity membership grade.

Note that the MF kinks that occur at zero membership grade have already been accounted for during the construction of the

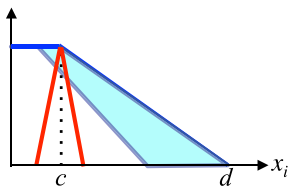

(a)

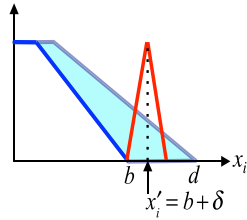

(c)

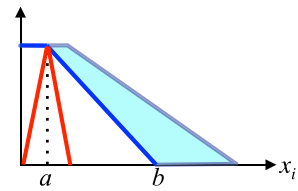

(b)

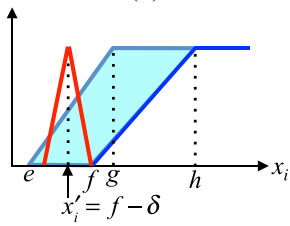

(d)
Fig. 8. Relative location of T1 FN to (a) UMF and (b)-(d) LMF.

first-order rule partitions and are therefore not involved in determining second-order rule partition lines.

Focusing next on a T1 NS IT2 fuzzy system, recall from (2), that in such a fuzzy system it is the interaction of the T1 FN with both the LMF and the UMF of an antecedent's FOU that contributes to the two max-star computations. Consequently, one has the following.

Definition 11: Appropriate locations for IT2 second-order rule partition lines in a T1 NS IT2 fuzzy system (that uses a triangle $\mathrm{T} 1 \mathrm{FN}$ ) are on the $x_{i}$-axis and are found by locating (a) where a UMF or an LMF has an MF kink (Definition 4) at unity membership grade [see Fig. 8(a), (b), and (d)], and (b) where all last encounters (Definition 6) of downward-sloping LMF lines occur [see Fig. 8(c)] and all first encounters (Definition 5) of upward-sloping LMF lines [see Fig. 8(d)], at the zero membership grade.

Item (a) should be obvious, from, e.g., Fig. 8(a) and (b), for which an IT2 second-order rule partition line occurs at the MF kinks $x_{i}=c$ and $x_{i}=a$, respectively, and Fig. 8(d), for which an IT2 second-order rule partition lines occur at the MF kinks $x_{i}=g$ and $x_{i}=h$. Note that if the FOU is a triangle such that the LMF and UMF meet at the same point when the membership grade is unity, then there will only be one IT2 second-order rule partition line at that common point.

Item (b) needs an explanation. First, note that the MF kinks that occur at a zero membership grade for the UMF have already been accounted for during the construction of the IT2 first-order rule partitions, and are therefore not involved in determining IT2 second-order rule partition lines.

Next, from Fig. 8(b) and (c), it should be clear that (analogous to the results in Fig. 7) for $x_{i} \in[a, b+\delta]$ the result of the maxmin computation is on the downward-sloping portion of the LMF, and, from Fig. 8(c), that for $x_{i} \geq b+\delta$ the result of the max-min computation is zero. However, as long as the UMF is not zero, the firing interval still contributes to the output, which is why the IT2 second-order rule partition line at the LMF kink has to be shifted from $b$ to $b+\delta$ [see Fig. 8(c)]. It is only for $x_{i} \geq d+\delta$ that the firing interval for the shoulder FOU no longer contributes to the output of the IT2 fuzzy system, but $x_{i}=d+\delta$ has already contributed an IT2 first-order rule partition line, so no new line is needed. 
TABLE III

Four-Step Procedure FOR ESTABLISHING T1 (IT2) SECOND-ORdER RULE PARTITION QUANTITIES FOR $X_{i}$, IN AN NS T1 (T1 NS IT2) FUZZY SYSTEM, ON A DRAWING OF ITS RESPECTIVE FIRST-ORDER RULE PARTITIONS

\begin{tabular}{|c|c|}
\hline Step & Description \\
\hline 1 & $\begin{array}{l}\text { Scan the axis of } x_{i} \text { and insert a dotted vertical line at all } \\
\text { appropriate locations for second-order rule partitions (Definitions } \\
10 \text { or 11). If any of these dotted lines occurs at a boundary of a T1 } \\
\text { (IT2) first-order rule partition, then do not draw such a vertical } \\
\text { dotted line. }\end{array}$ \\
\hline 2 & $\begin{array}{l}\text { The interval of real numbers between adjacent dotted vertical lines } \\
\text { or between a dotted line and a dashed (or dashed and dotted) line } \\
\text { is its T1 (IT2) second-order rule partition. }\end{array}$ \\
\hline 3 & $\begin{array}{l}\text { Each T1 (IT2) first-order rule partition has from zero to a finite } \\
\text { number of T1 (IT2) second-order rule partitions. Count them. }\end{array}$ \\
\hline 4 & $\begin{array}{l}\text { Count the total number of T1 (IT2) second-order rule partitions, } \\
\text { the total being } N_{T 1}^{2}\left(X_{i}\right)\left(N_{I T 2}^{2}\left(X_{i}\right)\right) \text {. }\end{array}$ \\
\hline
\end{tabular}

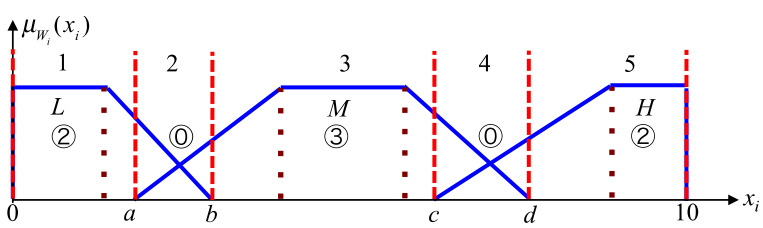

(a)

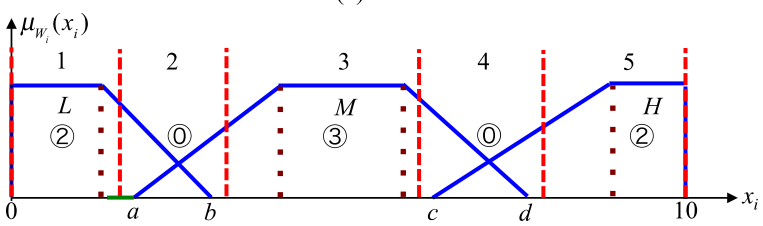

(b)

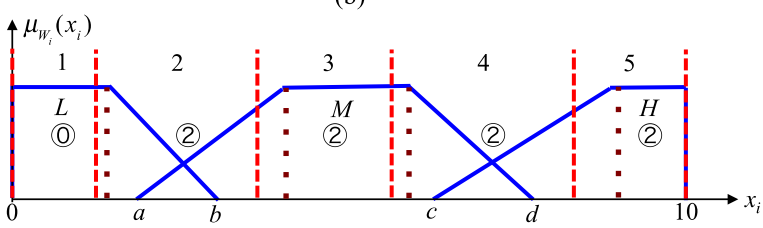

(c)

Fig. 9. Example 5 figures: circled numbers denote the number of $\mathrm{T} 1$ secondorder rule partitions in a respective $\mathrm{T} 1$ first-order rule partition (denoted $N_{T 1}^{2}\left(k_{x_{i}} \mid x_{i}\right)$ in [1] where $k_{x_{i}}=1, \ldots, 5$; see, also Table SM-V in SM). (a) S T1: $7 \mathrm{~T} 1$ second-order rule partitions. (b) NS T1 (4\% T1 FN): $7 \mathrm{~T} 1$ second-order rule partitions. (c) NS T1 (12\% T1 FN): 8 T1 second-order rule partitions.

A discussion similar to the one just given for Fig. 8(c) can also be given for Fig. 8(d), to explain why an IT2 second-order rule partition line occurs at $x_{i}=f-\delta$, and is left to the reader.

A formal four-step procedure for establishing T1 (IT2) second-order rule partition quantities for a single variable $x_{i}$, in an NS T1 (T1 NS IT2) fuzzy system, begins with a drawing of the respective T1 (IT2) first-order rule partitions, and is given in Table III. It is the extension of [12, Table V] from S to NS (see, also, Table SM-VI in SM).

Example 5: This is a continuation of Example 1. The results for Steps 1-3 of Table III are shown in Fig. 9(b) and (c) for 4\% and $12 \% \mathrm{~T} 1 \mathrm{FNs}$, respectively. The following are observed:

1) The fuzzy systems in Fig. 9(a) and (b) have the same total number of seven $\mathrm{T} 1$ second-order rule partitions although

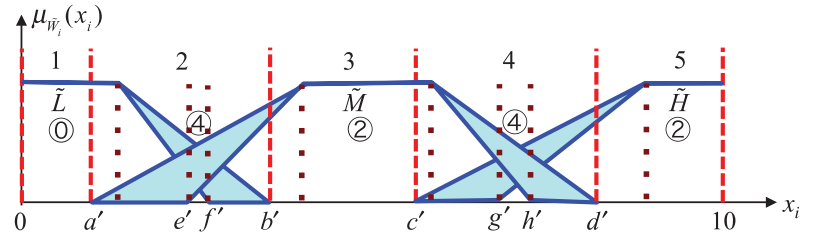

(a)

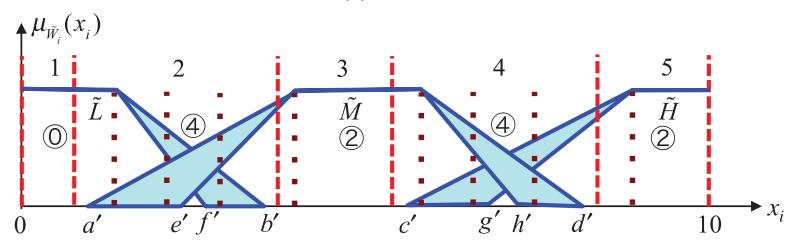

(b)

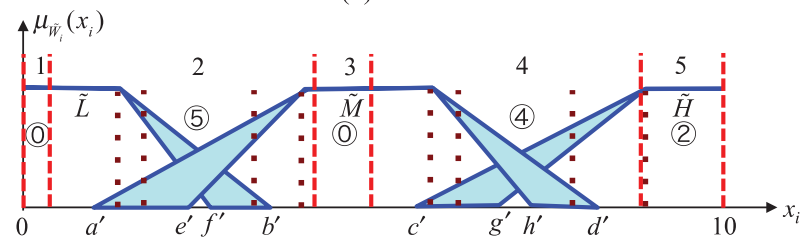

(c)

Fig. 10. Example 6 figures. Circled numbers denote the number of IT2 second-order rule partitions in a respective IT2 first-order rule partition (denoted $N_{I T 2}^{2}\left(k_{x_{i}} \mid x_{i}\right)$ in [11] where $k_{x_{i}}=1, \ldots, 5$; see, also Table SM-V in SM). (a) S IT2: 12 IT2 second-order rule partitions. (b) T1 NS IT2 (4\% T1 FN): 12 IT2 second-order rule partitions. (c) T1 NS IT2 (12\% T1 FN): 11 IT2 second-order rule partitions.

the sizes of some of those partitions are different for the two fuzzy systems.

2) The fuzzy system in Fig. 9(c) has eight T1 second-order rule partitions, which demonstrates that NS fuzzification can increase the number of second-order rule partitions.

Example 6: This is a continuation of Example 2. The results for Steps 1-3 of Table III are shown in Fig. 10(b) and (c). The following are observed:

1) Fuzzy systems in Fig. 10(a) and (b) have the same total number of 12 IT2 second-order rule partitions although the sizes of some of those partitions are different for the different fuzzy systems.

2) Fuzzy system in Fig. 10(c) has 11 IT2 second-order rule partitions, which demonstrates that NS fuzzification can also decrease the number of second-order rule partitions. See, also, Example SM-2 (Section VI of the SM) in which NS fuzzification does not change the number of IT2 second-order rule partitions.

Unlike Section III-A's definitive conclusions about the increase or decrease of the sizes of T1 (IT2) first-order rule partitions due to NS fuzzification, no such definitive conclusions can be drawn about the increase or decrease of the total number of T1 (IT2) second-order rule partitions due to NS fuzzification. This is also quite different from the definitive conclusions in [1] about the almost always increase (but no decrease) of the number of T1 (IT2) second-order rule partitions as one goes from an S T1 to an S IT2 fuzzy system, and is one demonstration of measurement uncertainty modeled as a T1 FN being quite 
different from antecedent MF uncertainty being modeled as an IT2 FS.

2) Second-Order Rule Partitions for $X_{1} \times X_{2}$ : The following definition is the extension of Definition $3 \mathrm{NS}$ from $X_{i}$ to $X_{1} \times X_{2}$ (see footnote 10$)$.

Definition 12: In an NS T1 (T1 NS IT2) fuzzy system, a T1 (IT2) second-order rule partition line of $X_{1} \times X_{2}$ occurs where the location of the value of either $x_{1}$ or $x_{2}$ at which the maximum occurs in (1) [(2)] $\left(x_{1}^{*}\right.$ or $\left.x_{2}^{*}\right)$ changes from one segment of an antecedent's MF (UMF or LMF) to another segment [within a T1 (IT2) first-order rule partition of $\left.X_{i}\right]$, where the slope of the latter segment differs from the slope of the former segment. ${ }^{14}$

A formal four-step procedure for establishing T1 (IT2) second-order rule partitions of $X_{1} \times X_{2}$ and related quantities begin with a drawing of the T1 (IT2) first-order rule partitions and proceed exactly as in [1, Table VI] (see also Table SM-VII in SM).

Example 7: This is a continuation of Example 3. The results of Steps 1-3 of Table SM-VII are shown in the six parts of Fig. 11. In each first-order rule partition, there are two numbers that are separated by a colon: the first is $N_{R}\left(k_{x_{1}}, k_{x_{2}}\right)$, and the second is $N_{*}\left(k_{x_{1}}, k_{x_{2}}\right)$; e.g., 2:3 indicates that two rules are fired in the first-order rule partition, and there are three second-order rule partitions in that first-order rule partition.

By adding all of the numbers that appear to the right of the colons in each of the Fig. 11 figures, one obtains the total numbers of second-order rule partitions that are stated in the captions to those figures. Observe that the partitions for the $4 \%$ $\mathrm{T} 1 \mathrm{FN}$ are somewhat different from those for $\mathrm{S}$ fuzzification (although it may be difficult to discern differences between Fig. 11(a) and (c) and Fig. 11(b) and (d), due to their reduced sizes, they are different, as can be more readily observed when Fig. 9(a) and (b) are compared, and when Fig. 10(a) and (b) are compared); however, the partitions for the $12 \% \mathrm{~T} 1 \mathrm{FN}$ are very different.

Example 8: Fig. 12 depicts control surfaces for the six fuzzy systems in Fig. 11; they used max-min inference (control surfaces that use max-product inference are in Section VIII of the $\mathrm{SM}$ ), center-of-sets (COS) defuzzification for the T1 fuzzy systems and COS type reduction (TR) for the IT2 fuzzy systems; numerical information about the nine rules and their consequents are given in Section VIII in the SM. From the control surfaces, the following are observed.

1) When one compares each $\mathrm{T} 1$ control surface in the lefthand column with its respective IT2 control surface in the right-hand column, it is clear that the combination of NS fuzzification and IT2 FSs leads to smoother control surfaces, which means a better interpolation of fired rules, i.e., a small change in an input results in a smaller change in the output and hence to better performance.

2) The flat plateaus occur in the nine first-order rule partitions in which only one rule fires and are due to COS defuzzification or COS TR, for which the control output is always a constant; for the T1 fuzzy system, this equals the COG of the consequent FS, and for the IT2 fuzzy system

\footnotetext{
${ }^{14}$ In general, the T1 FNs for $x_{1}$ and $x_{2}$ can be different.
}

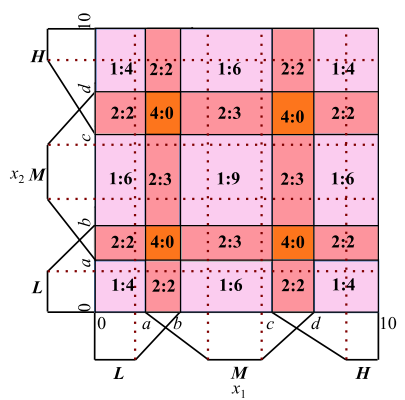

(a)

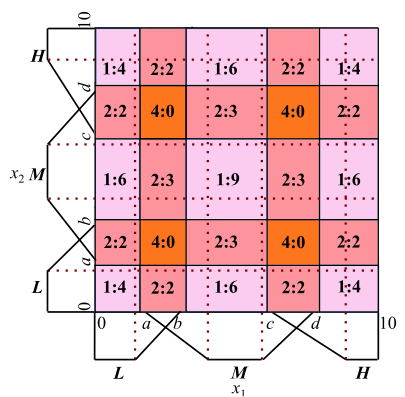

(c)

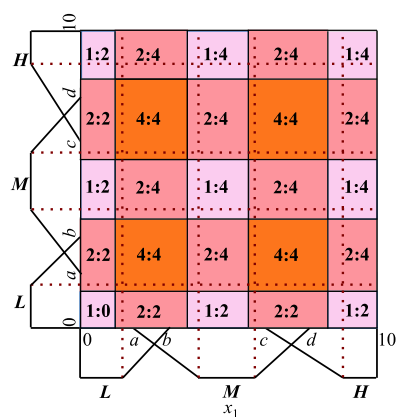

(e)

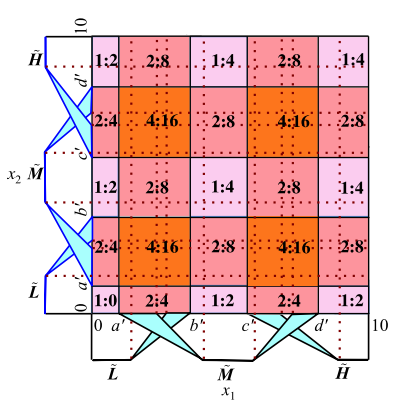

(b)

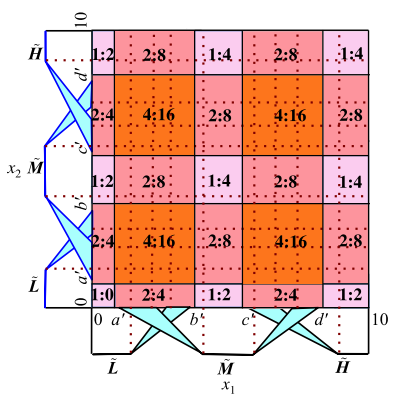

(d)

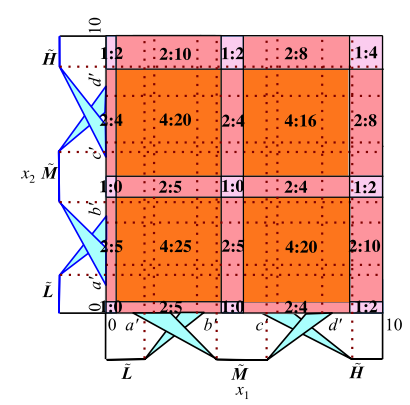

(f)
Fig. 11. Example 7 figures. Each figure is the T1 (IT2) second-order rule partition diagram for its corresponding figure that is in Fig. 6. In the captions to each figure, "partitions" is short for "second-order rule partitions." (a) S T1: 77 T1 partitions. (b) S IT2: 167 IT2 partitions. (c) NS T1 (4\% T1 FN): 77 T1 partitions. (d) T1 NS IT2 (4\% T1 FN): 167 IT2 partitions. (e) NS T1 (12\% T1 FN): 80 T1 partitions. (f) T1 NS IT2 (12\% T1 FN): 165 IT2 partitions.

this equals the average of the left and right end-points of the centroid of the consequent IT2 FS.

3) Second-Order Rule Partitions for $X_{1} \times X_{2} \times \cdots \times X_{p}$ :

Definition 13: In an NS T1 (T1 NS IT2) fuzzy system, a T1 (IT2) second-order rule partition line of $X_{1} \times X_{2} \times \cdots \times X_{p}$ occurs where the location of the value of either $x_{1}$ or $x_{2}$ or ... or $x_{p}$ at which the maximum occurs in (1) [(2)] ( $x_{1}^{*}$ or $x_{2}^{*} \ldots$ or $x_{p}^{*}$ ) changes from one segment of an antecedent's MF (UMF or LMF) to another segment [within a T1 (IT2) first-order rule partition of $X_{i}$ ], where the slope of the latter segment differs from the slope of the former segment.

A formula for the total number of T1 (IT2) second-order rule partitions of $X_{i}, N_{*}^{2}\left(X_{i}\right)$ is

$$
N_{*}^{2}\left(X_{i}\right)=\sum_{k_{i}=1}^{N_{*}^{1}\left(X_{i}\right)} N_{*}^{2}\left(k_{i} \mid x_{i}\right) .
$$




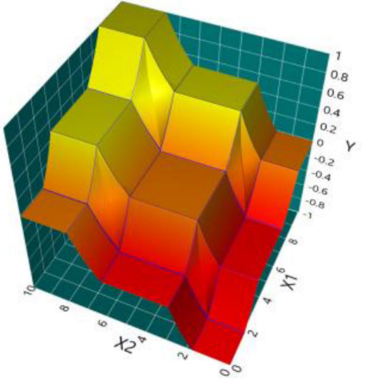

(a)

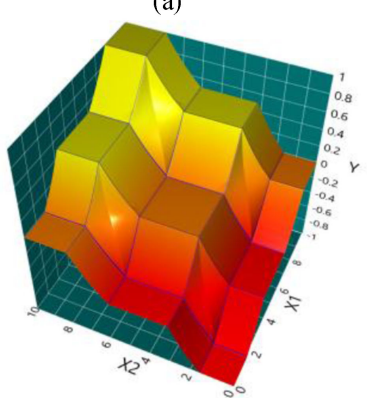

(c)

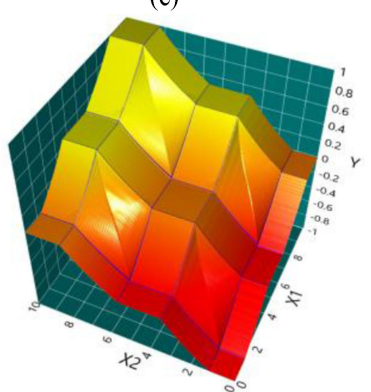

(e)

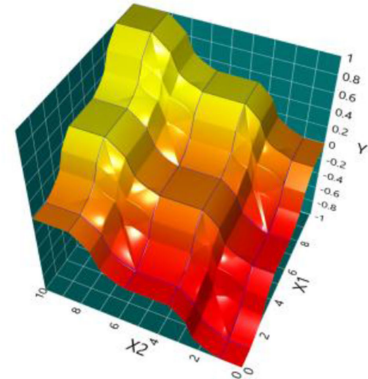

(b)

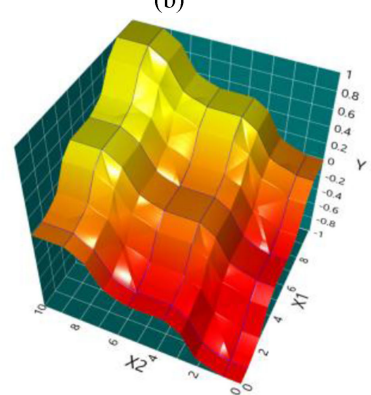

(d)

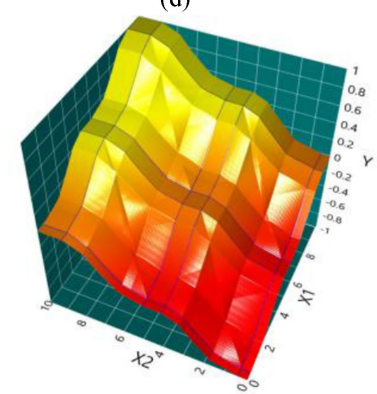

(f)
Fig. 12. Example 8 figures. Each figure is a T1 (IT2) control surface for its corresponding figure that is in Fig. 11. (a) S T1. (b) S IT2. (c) NS T1 (4\% T1 FN). (d) T1 NS IT2 (4\% T1 FN). (e) NS T1 (12\% T1 FN). (f) T1 NS IT2 $(12 \% \mathrm{~T} 1 \mathrm{FN})$.

In (5), $N_{*}^{2}\left(k_{i} \mid x_{i}\right)$ [the total number T1 (IT2) second-order rule partitions within the $k_{i}$ th T1 (IT2) first-order rule partition of $X_{i}$ ] are obtained by counting (see Table III, Step 3). A formula for the total number of T1 (IT2) second-order rule partitions of $X_{1} \times X_{2} \times \cdots \times X_{p}, N_{*}^{2}\left(X_{1}, \ldots, X_{p}\right)$ is

$$
\begin{aligned}
N_{*}^{2}\left(X_{1}, \ldots, X_{p}\right)= & \prod_{j=1}^{p}\left[N_{*}^{2}\left(X_{j}\right)+Z\left(X_{j}\right)\right] \\
& -\prod_{j=1}^{p} Z\left(X_{j}\right) \\
Z\left(X_{j}\right)= & \sum_{k_{j}=1}^{N_{*}^{1}\left(X_{j}\right)} \xi\left(k_{j} \mid x_{j}\right) \\
\xi\left(k_{j} \mid x_{j}\right)= & \left\{\begin{array}{lll}
0 & \text { if } & N_{*}^{2}\left(k_{j} \mid x_{j}\right) \neq 0 \\
1 & \text { if } & N_{*}^{2}\left(k_{j} \mid x_{j}\right)=0 .
\end{array}\right.
\end{aligned}
$$

Note that (6) is analogous to [1, eq. (13)] and that the explanation and reason that are given for the appearance of $Z\left(X_{j}\right)$

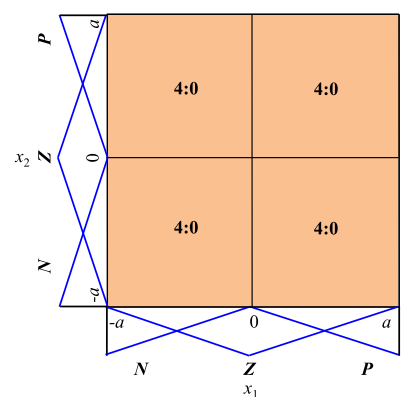

(a)

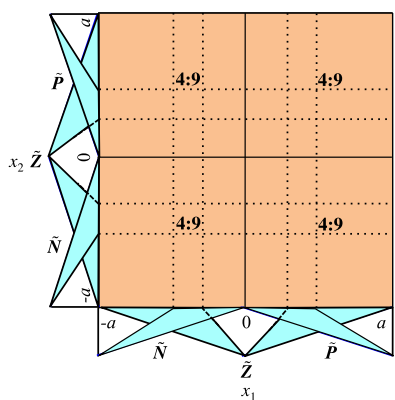

(b)
Fig. 13. (a) and (b) First and second-order rule partitions of $X_{1} \times X_{2}$ for Example 5 in [1]. Regarding $a: b, a$ denotes the number of same rules fired in the T1 (IT2) first-order rule partition, and $b$ denotes the number of T1 (IT2) second-order rule partitions within that T1 (IT2) first-order rule partition (count them). (a) S T1 fuzzy system. (b) S IT2 fuzzy system.

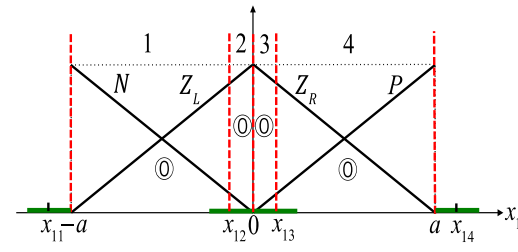

(a)

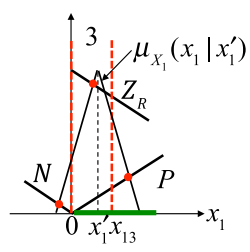

(b)
Fig. 14. (a) T1 first- and second-order rule partitions of $X_{1}$ for the example in Fig. 13(a), and for the $12 \% \mathrm{~T} 1 \mathrm{FN} ; Z_{L}\left(Z_{R}\right)$ denotes left (right) segment of Z. (b) Blowup of Partition 3 for $x_{1}^{\prime} \in\left[0, x_{13}\right]$ in which the three filled-in red circles demonstrate a nonzero value for the max-min computation between $\mu_{X_{1}}\left(x_{1} \mid x_{1}^{\prime}\right)$ and $\mu_{N}\left(x_{1}\right), \mu_{Z_{R}}\left(x_{1}\right)$ and $\mu_{P}\left(x_{1}\right)$, indicating three rules fire when $x_{1}^{\prime} \in\left[0, x_{13}\right]$.

in [1, eq. (13)] are the same for why $Z\left(X_{j}\right)$ appears in our (6) (see also Section V in the SM).

\section{New PHENOMENON: Rule CROSSOVER}

Fig. 13 is taken from [1, Example 5], for which the S T1 fuzzy system has no T1 second-order rule partitions whereas the S IT2 fuzzy system has 36 of them. Consequently, it is stated [1] that “... although the T1 and IT2 fuzzy systems have exactly the same number of first-order rule partitions (four) ... there is no further sculpting of the T1 fuzzy system, whereas there is much further sculpting of the IT2 fuzzy system."

Instead of immediately presenting the NS versions of Fig. 13(a) and (b), we return first to the T1 first-and second-order partition diagram just for $x_{1}$ in the NS situation (the diagram for $x_{2}$ is exactly the same); it is obtained from Table I and is depicted in Fig. 14(a) for the 12\% T1 FN (similar results hold for the $4 \% \mathrm{~T} 1 \mathrm{FN}$ ). This figure needs some explanation.

Observe there are four T1 first-order rule partitions 1-4. One might argue that there should only be two such partitions, obtained by stretching $x_{1}=0$ (the right-end boundary of the T1 partition $[-a, 0]$ for the $\mathrm{S}$ T1 fuzzy system) to the right until it reaches $x_{1}=x_{13}$ and by also stretching $x_{1}=0$ (also the leftend boundary of the $\mathrm{T} 1$ partition $[0, a]$ for the $\mathrm{S} \mathrm{T} 1$ fuzzy system) to the left until it reaches $x_{1}=x_{12}$; but, this is incorrect because doing both of these would lead to two overlapping regions where 
the overlap is $\left[x_{11}, x_{12}\right]$, which would mean that $x_{1} \in\left[x_{11}, x_{12}\right]$ would exist simultaneously in two T1 first-order rule partitions, something that violates the meaning of a mathematical partition.

To understand this better, one can examine what the antecedents of the two fired-rules are in each of the four T1 first-order rule partitions. From the MFs in Fig. 14(a) [see, also Fig. 14(b) for line 3 of (9)], one obtains

$$
\begin{cases}x_{1} \in\left[-a, x_{12}\right]: & \left\{\operatorname{IF} x_{1} \text { is } N, \operatorname{IF} x_{1} \text { is } Z_{L}\right\} \\ x_{1} \in\left[x_{12}, 0\right]: & \left\{\operatorname{IF} x_{1} \text { is } N, \operatorname{IF} x_{1} \text { is } Z_{L}, \operatorname{IF} x_{1} \text { is } P\right\} \\ x_{1} \in\left[0, x_{13}\right]: & \left\{\operatorname{IF} x_{1} \text { is } N, \operatorname{IF} x_{1} \text { is } Z_{R}, \operatorname{IF} x_{1} \text { is } P\right\} \\ x_{1} \in\left[x_{13}, a\right]: & \left\{\operatorname{IF} x_{1} \text { is } P, \operatorname{IF} x_{1} \text { is } Z_{R}\right\} .\end{cases}
$$

Observe that (9) demonstrates that different combinations of rules are fired in $x_{1} \in\left[x_{12}, 0\right]$ and $x_{1} \in\left[0, x_{13}\right]$, and so they are indeed legitimate $\mathrm{T} 1$ first-order rule partitions.

Amazingly, NS fuzzification leads to two rules: IF $x_{1}$ is $P$ in line 2 [compare lines 1 and 2 in (9)] and IF $x_{1}$ is $N$ in line 3 [compare lines 3 and 4 in (9)] - crossover rules - that can never occur in an S fuzzy system, and it is partition theory that has revealed this.

The NS versions of the two figures in Fig. 13 are shown in Fig. 15. Observe that, whereas the S T1 fuzzy system in Fig. 13(a) has four T1 first-order rule partitions and no T1 second-order rule partitions, the NS T1 fuzzy system in Fig. 15(a) has $16 \mathrm{~T} 1$ first-order rule partitions but still no T1 second-order rule partition. This demonstrates a new phenomenon for a T1 fuzzy system that NS fuzzification can increase the number of its T1 first-order rule partitions.

Observe, also that, whereas the S IT2 fuzzy system in Fig. 13(b) has four IT2 first-order rule partitions and 36 IT2 secondorder rule partitions, the T1 NS IT2 fuzzy system in Fig. 15(b) has 16 IT2 first-order rule partitions and 60 IT2 second-order rule partitions. This demonstrates a new phenomenon for an IT2 fuzzy system that NS fuzzification can simultaneously increase the numbers of both its IT2 first-order and second-order rule partitions. See, also Example SM-3 in Section VII of the SM.

Example 9: This example is a continuation of Examples 1,5 7, and 8, for a T1 fuzzy system, and Examples 2, 6, 7, and 8 for an IT2 fuzzy system, to the case of a $24 \%$ T1 FN. Interestingly, no rule-crossover occurs for the T1 fuzzy system [see Fig. 16(a)], but it does occur for the IT2 fuzzy system (observe, e.g., in Fig. 16(b), that $b^{\prime}+\delta>c^{\prime}-\delta$ ). In this example, rule-crossover reduces the number of first (second)-order rule partitions from five [11 in Fig. 10(c)] to four (10). Although the size of the first-order rule partition \#2 [e.g., in Fig. 16(b)], due to crossover is small, it would be larger if the support of the T1 FN was larger than $24 \%$. Finally, observe that the control surfaces for the $24 \%$ T1 FN in Fig. 16(g) and (h) are noticeably different from the ones for 12\% T1 FM in Fig. 12(e) and (f).

The two examples in this section suggest that more research is needed about how to overlap MFs (FOUs) so as to obtained increased or decreased numbers of first (second)-order rule partitions, as well as whether or not rule crossover is good or bad.

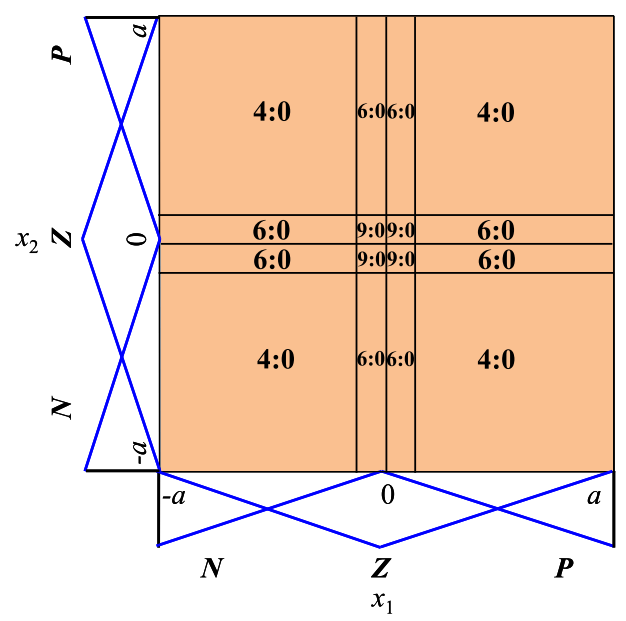

(a)

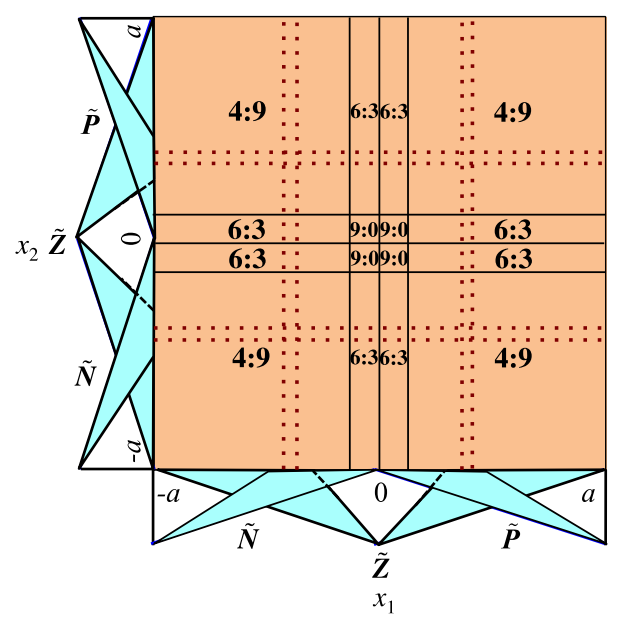

(b)

Fig. 15. (a), (b) 12\% T1 FN NS versions of Fig. 13(a) and (b), respectively.

\section{WHICH RULES FIRE?}

In an S T1 (IT2) fuzzy system, it is straightforward to enumerate which rules fire in a specific first-order rule partition, by examining which MFs (FOUs) are intersected by a vertical line drawn at $x=x^{\prime}$. Because this is so easy to do, and no ambiguities can occur, there was no major concern in [1] or [2]. Unfortunately, the same is not true in an NS T1 (IT2) fuzzy system because it is no longer a vertical line at $x=x^{\prime}$ that establishes which rules are fired in a specific T1 (IT2) first-order rule partition. Instead, it is a T1 FN that is located about $x=x^{\prime}$ that does this. An illustration of this has been given in (9) for the T1 FSs in Fig. 14.

If one is actually interested to know which rules fire in a specific T1 (IT2) first-order rule partition for an NS T1 (T1 NS IT2) fuzzy system, one must provide this as additional information for each such partition. We have chosen not to do this for our examples because the purpose of this paper is to better understand the performance potential of an NS fuzzy system over an S fuzzy system, and one does not need to know which rules fire in a specific T1 (IT2) first-order rule partition in order to accomplish this. 


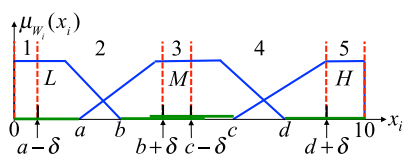

(a)

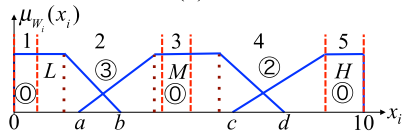

(c)

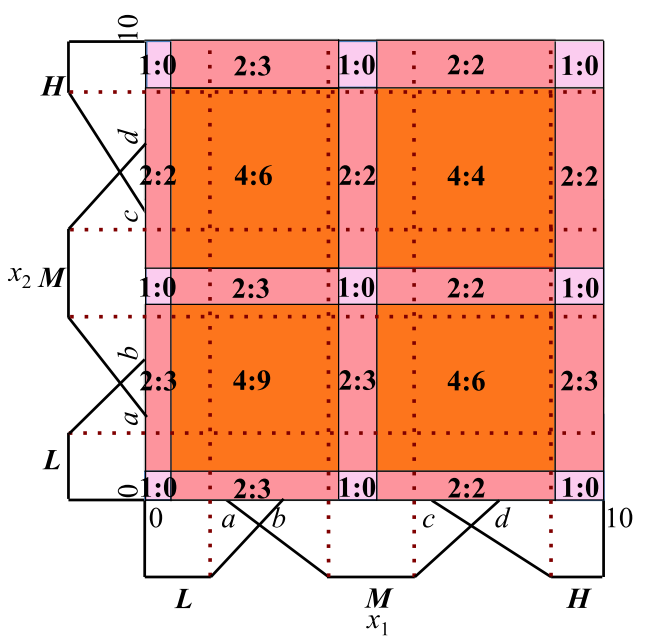

(e)

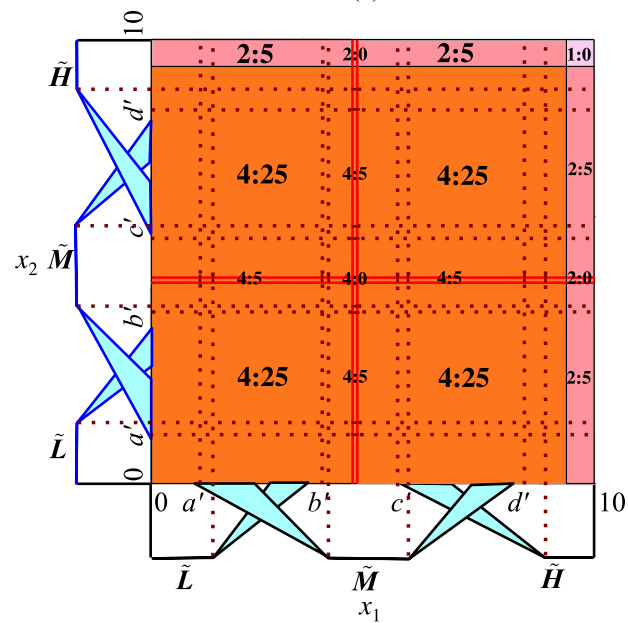

(f)

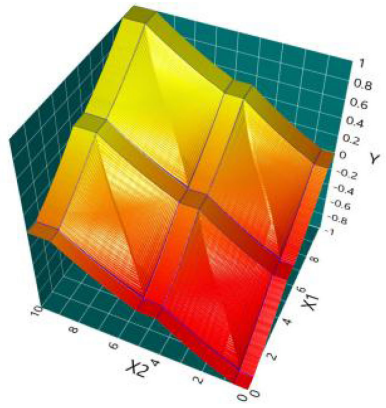

(g)

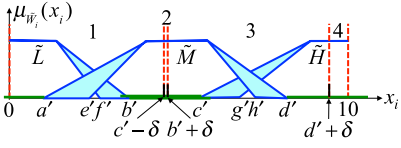

(b)

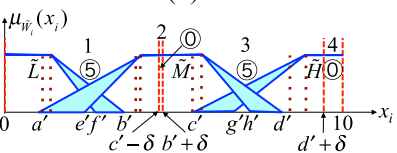

(d)

\section{CONCLuSion AND Future RESEARCH}

The purpose of this paper has been to better understand the performance potential of an NS fuzzy system over an S fuzzy system. The approach to doing this has been to extend [1] from $\mathrm{S}$ to NS fuzzification. ${ }^{15}$ The paper's main conclusions are uncertainties about measurements, modeled by NS fuzzification:

1) Fire more rules more often (regardless of the nature of the fuzzy system) manifested by a reduction in the sizes of T1 and IT2 first-order rule partitions for those partitions associated with the firing of a smaller number of rules, and an increase in the sizes of T1 and IT2 first-order rule partitions for those partitions associated with the firing of a larger number of rules- the coarse sculpting of the state space.

2) It may lead to an increase or decrease in the number of T1 and IT2 first-order rule partitions, and to some partitions that contain rule combinations that can never occur for $\mathrm{S}$ fuzzification - a new rule crossover phenomenondiscovered by using partition theory.

3) It may lead to a decrease, the same number, or an increase in the number of T1 and IT2 second-order rule partitionsthe fine sculpting of the state space - all of which are very system dependent.

4) It leads to better control surfaces with smoother transitions between the various areas of the control surface, i.e, a small change in the input results in smaller changes in the output and hence to better system performance.

The author's conjecture is that it is the additional control of the coarse sculpting of the state space, accomplished by prefiltering and the max-min (or max-product) composition, which provides an NS T1 or IT2 fuzzy system with the potential to outperform an ST1 or IT2 fuzzy system when measurements are uncertain.

Some open research questions and extensions to this paper are as follows:

1) prove the just-stated conjecture using the framework of rule partitions for NS T1 (IT2) fuzzy systems;

2) extend the paper's results to other kinds of FNs, e.g., trapezoidal;

3) extend the paper's results to NS general T2 fuzzy systems;

4) develop new geometric design methods that are based on first- and second-order rule partitions (e.g., analyze where in the state-space largest errors occur and then alter MF (FOU) shapes in those regions so that more first and/or second-order rule partitions occur in them);

5) establish a methodology for overlapping MFs (FOUs) so as to obtain and establish if rule crossover is good or bad;

6) study whether or not NS fuzzification can improve the performance of a fuzzy logic controller (e.g., fuzzy proportional-integral-derivative-FPID—controller) by virtue of its new rule crossover phenomenon;

7) extend the paper's results to similarity-based inference engines for NS fuzzification [30], [33]-[37].

Fig. 16. Example 9, 24\% T1 FN NS T1 [(a), (c), (e), (g)] and T1 NS IT2 [(b), (d), (f), (h)] rule partition figures. (a) Five first-order rule partitions. (b) Four first-order rule partitions. (c) Five second-order rule partitions. (d) Ten secondorder rule partitions. (e) 25 first-order and 55 second-order rule partitions. (f) 16 first-order and 140 IT2 second-order rule partitions. (g) NS T1 control surface. (h) T1 NS IT2 control surface.

\footnotetext{
${ }^{15}$ An online site has been developed that lets the reader replicate the examples of this paper as well as apply the theory to other examples of two-input one output fuzzy systems. It is: http://fuzzypartitions.com/
} 


\section{ACKNOWLEDGMENT}

The authors would like to thank the reviewers of this paper for their insightful comments and suggestions.

\section{REFERENCES}

[1] J. M. Mendel, "Explaining the performance potential of rule-based fuzzy systems as a greater sculpting of the state space," IEEE Trans. Fuzzy Syst., vol. 26, no. 4, pp. 2362-2373, Aug. 2018.

[2] J. M. Mendel, "Comparing the performance potentials of interval and general type-2 rule-based fuzzy systems in terms of sculpting the state space," IEEE Trans. Fuzzy Syst., vol. 27, no. 1, pp. 58-71, Jan. 2019.

[3] G. C. Mouzouris and J. M. Mendel, "Nonsingleton fuzzy logic systems," in Proc. 3rd IEEE Conf. Fuzzy Syst., Orlando, FL, USA, Jun. 1994, pp. $456-461$.

[4] G. C. Mouzouris and J. M. Mendel, "Nonlinear time-series analysis with nonsingleton fuzzy logic systems," in Proc. IEEE/IAFE Conf. Comput. Intell. Financial Eng., New York City, NY, USA, Apr. 1995, pp. $47-56$.

[5] G. C. Mouzouris and J. M. Mendel, "Nonlinear predictive modeling using dynamic nonsingleton fuzzy logic systems," in Proc. IEEE Conf. Fuzzy Syst., New Orleans, LA, USA, Sep. 8-10, 1996, pp. 1217-1223.

[6] G. C. Mouzouris and J. M. Mendel, "Nonsingleton fuzzy logic systems: theory and application," IEEE Trans. Fuzzy Syst., vol. 5, no. 1, pp. 56-71, Feb. 1997.

[7] G. C. Mouzouris and J. M. Mendel, "Dynamic nonsingleton fuzzy logic systems for nonlinear modeling," IEEE Trans. Fuzzy Syst., vol. 5, no. 2 , pp. 199-208, May 1997.

[8] Q. Liang and J. M. Mendel, "Interval type-2 fuzzy logic systems: Theory and design," IEEE Trans. Fuzzy Syst., vol. 8, no. 5, pp. 535-550, Oct. 2000

[9] J. M. Mendel, Uncertain Rule-Based Fuzzy Logic Systems: Introduction and New Directions. Upper Saddleback River, NJ, USA: Prentice-Hall, 2001.

[10] J. M. Mendel, Uncertain Rule-Based Fuzzy Systems: Introduction and New Directions, 2nd ed. Cham, Switzerland: Springer, 2017.

[11] Z. H. Akpolat, "Nonsingleton fuzzy logic control of a DC motor," J. Appl. Sci., vol. 5, pp. 887-891, 2005.

[12] A. Simon and G. T. Flowers, "Disturbance attenuation using nonsingleton fuzzy logic," in Proc. ASME Int. Des. Eng. Tech. Conf. Comput. Inf. Eng. Conf., Long Beach, CA, USA, vol. 1, Sep. 2005, pp. 97-106.

[13] R. K. Nowicki and J. T. Staarczewski, "On nonsingleton fuzzification with DCOG defuzzification," in Artificial Intelligence and Soft Computing, ICAISC 2010, (Lecture Notes in Computer Science), L. Rutkowski, R. Scherer, R. Tadeusiewicz, L. A. Zadeh, and J. M. Zurada, Eds. Berlin, Germany: Springer, 2010, vol. 6113, pp. 168-174.

[14] G. M. Mendez and M.A. Hernandez, "Interval type-1 nonsingleton type-2 fuzzy logic systems are type-2 adaptive neuro-fuzzy inference systems," Int. J. Reason.-based Intell. Syst., vol. 2, pp. 95-99, 2010.

[15] A. B. Cara, I. Rojas, H. Pomares, C. Wagner, and H. Hagras, "On comparing nonsingleton type-1 and singleton type-2 fuzzy controllers for a nonlinear servo system," in Proc. IEEE Symp. Adv. Type- 2 Fuzzy Logic Syst., 2011, pp. 126-133.

[16] T. W. Chua and W. W. Tan, "Nonsingleton genetic fuzzy logic system for arrhythmias classification," Eng. Appl. Artif. Intell., vol. 24, pp. 251-259, Mar. 2011.

[17] M. de los Angeles Hernandez, P. Melin, G. M. Mendez, O. Castillo and I. L.-Juarez, "A hybrid learning method composed by the orthogonal least-squares and the back-propagation learning algorithms for interval A2-C1 type-1 nonsingleton type-2 TSK fuzzy logic systems," Soft Comput., vol. 19, no. 3, pp. 661-678, 2015.

[18] C. Fu, A. Sarabakha, E. Kayacan, C. Wagner, R. John, and J. M. Garibaldi, "Input uncertainty sensitivity enhanced nonsingleton fuzzy logic controllers for long-term navigation of quadrotor UAVs," IEEE/ASME Trans. Mechatronics, vol. 23, no. 2, pp. 725-734, Apr. 2018.
[19] D. Hidalgo, P. Melin, and J. R. Castro, "Nonsingleton interval type-2 fuzzy systems as integration methods in modular neural networks used genetic algorithms to design," in Nature-Inspired Design of Hybrid Intelligent Systems (Studies in Computational Intelligence), vol. 667, P. Melin, O. Castillo, and J. Kacprzyk, Eds. Cham, Switzerland: Springer, 2017, pp. 821-838.

[20] R. Martínez-Soto, O. Castillo, and J. R. Castro, "Genetic algorithm optimization for type-2 nonsingleton fuzzy logic controllers," in Recent Advances on Hybrid Approaches for Designing Intelligent Systems (Studies in Computational Intelligence), vol. 547, O. Castillo, P. Melin, W. Pedrycz, and J. Kacprzyk, Eds. Cham, Switzerland: Springer, 2014, pp. 3-18.

[21] G. M. Mendez, O. Castillo, R. Colas, and H. Moreno, "Finishing mill strip gage setup and control by interval type-1 nonsingleton type-2 fuzzy logic systems," Appl. Soft Comput., vol. 24, pp. 900-911, Nov. 2014.

[22] G. M. Mendez and M. A. Hernandez, "Hybrid learning mechanism for interval A2-C1 type-2 nonsingleton type-2 Takagi-Sugeno-Kang fuzzy logic system,' Inf. Sci., vol. 220, pp. 149-169, 2013.

[23] A. Pourabdollah, R. John, and J. M. Garibaldi, "A new dynamic approach for nonsingleton fuzzification in noisy time-series prediction," in Proc. IEEE Int. Conf. Fuzzy Syst., Naples, Italy, Jul. 2017, pp. 1-7.

[24] A. Pourabdollah, C. Wagner, J. Aladi, and J. Garibaldi, "Improved uncertainty capture for nonsingleton fuzzy systems," IEEE Trans. Fuzzy Syst., vol. 24, no. 6, pp. 1513-1524, Dec. 2016.

[25] A. Pourabdollah, C. Wagner, and J. Aladi, "Changes under the hood-a new type of nonsingleton fuzzy logic system," in Proc. IEEE Int. Conf Fuzzy Syst., Istanbul, Turkey, Aug. 2-5, 2015, pp. 1-8.

[26] G. Ruiz-Garcia, H. Pomares, I. Rojas, and H. Hagras, "The nonsingleton fuzzification operation for general forms of interval type-2 fuzzy logic systems," in Proc. IEEE Int. Conf. Fuzzy Syst., Naples, Italy, Jul. 2017, pp. $1-7$.

[27] N. Sahab and H. Hagras, "A type-2 nonsingleton type-2 fuzzy logic system to handle linguistic and numerical uncertainties in real world environments," in Proc. IEEE Symp. Adv. Type-2 Fuzzy Logic Syst., 2011, pp. $110-117$.

[28] N. Sahab and H. Hagras, "Adaptive nonsingleton type-2 fuzzy logic systems: a way forward for handling numerical uncertainties in real world applications," Int. J. Comput. Commun. Control, vol. 6 no. 3, pp. 503-529, Sep. 2011.

[29] A. Tellez-Velazquez and R. Cruz-Baraosa, "A CUDA-streams inference machine for non-singleton fuzzy systems," Concurrency Comput., Pract. Experience, vol. 30. no. 8, p. e4382, Apr. 2018.

[30] C. Wagner, A. Pourabdollah, J. McCulloch, R. John, and J. Garibaldi, "A similarity-based inference engine for nonsingleton fuzzy logic systems," in Proc. IEEE Int. Conf. Fuzzy Syst., 2016, Vancouver, BC, Canada, pp. 316-323.

[31] M. H. F. Zarandi, M. Yalinezhaad, and I. B. Turksen, "Two factors high order non singleton type-1 and interval type-2 fuzzy systems for forecasting time series with genetic algorithm," Adv. Time Series Forecasting, vol. 2, pp. 37-75, 2017.

[32] J. M. Mendel, "Fuzzy logic system for engineering: A tutorial," IEEE Proc., vol. 83, no. 3, pp. 345-377, Mar. 1995.

[33] H. Bustince, "Indicator of inclusion grade for interval-valued fuzzy sets. Application to approximate reasoning based on interval-valued fuzzy sets," Int. J. Approx. Reason., vol. 23, no. 3, pp. 137-207, 2000.

[34] J. M. Mendel and D. Wu, Perceptual Computing: Aiding People in Making Subjective Judgments. Hoboken, NJ, USA: Wiley-IEEE Press, 2010

[35] S. Raha, N. Pal, and K. Ray, "Similarity-based approximate reasoning: Methodology and application," IEEE Trans. Syst. Man Cybern., A, vol. 32, no. 4, pp. 541-547, Jul. 2002.

[36] D. Wu and J. M. Mendel, "Perceptual reasoning for perceptual computing: A similarity-based approach," IEEE Trans. Fuzzy Syst., vol. 17, no. 6, pp. 1397-1411, Dec. 2009

[37] S. Yeung and E. C. C. Tsang, "A comparative study on similarity-based fuzzy reasoning methods," IEEE Trans. Syst. Man Cybern., B, vol. 27, no. 6, pp. 216-227, Apr. 1997. 